\title{
Cause-specific mortality of low and selective intermediate-risk prostate cancer patients with active surveillance or watchful waiting
}

\author{
Xiangkun Wu ${ }^{1,2 \#}$, Daojun $\mathrm{Lv}^{1,2 \#}$, Md Eftekhar ${ }^{3}$, Chao Cai ${ }^{1,2}$, Zhijian Zhao ${ }^{1,2}$, Di Gu ${ }^{1,2}$, Yongda Liu ${ }^{1,2} \wedge$ \\ ${ }^{1}$ Department of Urology, Minimally Invasive Surgery Center, The First Affiliated Hospital of Guangzhou Medical University, Guangzhou, \\ China; ${ }^{2}$ Guangdong Key Laboratory of Urology, Guangzhou Institute of Urology, Guangzhou, China; ${ }^{3}$ Department of Family Medicine, CanAm \\ International Medical Center, Shenzhen, China \\ Contributions: (I) Conception and design: Y Liu, D Gu; (II) Administrative support: Y Liu; (III) Provision of study materials or patients: None; (IV) \\ Collection and assembly of data: C Cai, Z Zhao, M Eftekhar; (V) Data analysis and interpretation: X Wu, D Lv; (VI) Manuscript writing: All authors; \\ (VII) Final approval of manuscript: All authors. \\ \#These authors contributed equally to this work. \\ Correspondence to: Prof. Yongda Liu, Di Gu, PhD. Minimally Invasive Surgery Center, The First Affiliated Hospital of Guangzhou Medical University, \\ Kangda Road 1\#, Haizhu District, Guangzhou 510230, China. Email: 13719007083@163.com, di.gu@doctors.org.uk.
}

Background: Active surveillance or watchful waiting (AS/WW) is increasingly being used as an alternative strategy to radical prostatectomy or radiation therapy for appropriately selected patients with prostate cancer (PCa). However, the prognosis of low-risk and selective intermediate-risk PCa patients after AS/WW is poorly defined. In this study we reviewed the patients registered in the Surveillance, Epidemiology, and End Results (SEER) Program to establish a competing risk nomogram for the prediction of prostate cancerspecific mortality (PCSM).

Methods: The information of patients undergoing AS/WW in the SEER program from 2004 to 2015 was obtained. All patients were ISUP (International Society of Urological Pathology) grade 1 or 2 PCa and also fulfilled the National Comprehensive Cancer Network's definition of low-risk PCa [prostate specific antigen (PSA) $<10 \mathrm{ng} / \mathrm{mL}$ and $\mathrm{cT} 2 \mathrm{aN} 0 \mathrm{M} 0$ or less)]. A competing risk nomogram was used to analyze the association of tumor characteristics with PCSM and non-PCSM among the PCa patients with AS/WW. All cases were randomly divided into a training cohort and a validation cohort (1:1). A competing risk nomogram was constructed to predict PCSM in PCa patients with AS/WW. The performance of the PCSM nomogram was evaluated using the concordance index (C-index) and calibration curve.

Results: A total of 30,538 PCa patients were identified as low risk or selective intermediate risk with AS/ WW. The 10-year cumulative incidence of death from prostate cancer and death from other cause were 2.8\% (95\% CI: 2.4-3.1\%) and 19.3\% (95\% CI: 17.8-20.5\%), respectively. Variables associated with PCSM included age, marital status, PSA, and ISUP grade. The PCSM nomogram had a good performance in both the training and validation cohorts, with a C-index of 0.744 (95\% CI: $0.700-0.781, \mathrm{P}<0.001)$ and $0.738(95 \%$ CI: $0.700-0.777, \mathrm{P}<0.001)$, respectively.

Conclusions: Overall, the prognosis was favorable for the low- and selective intermediate-risk PCa patients with AS/WW. The competing risk nomogram yielded a good performance in identifying subgroups of patients with a higher risk of PCSM and potential candidates for AS/WW.

Keywords: Active surveillance; watchful waiting; prostate cancer-specific mortality; a competing risk nomogram

Submitted Jun 11, 2020. Accepted for publication Oct 20, 2020.

doi: $10.21037 /$ tau-20-994

View this article at: http://dx.doi.org/10.21037/tau-20-994

^ ORCID: Xiangkun Wu, 0000-0002-4952-9170; Yongda Liu, 0000-0003-2032-1314. 


\section{Introduction}

Prostate cancer $(\mathrm{PCa})$ remains the most common male cancer and is among the leading cause of cancer-related deaths in men in industrialized countries. In 2017 , approximately 1.3 million men were diagnosed with $\mathrm{PCa}$ worldwide and there were 416,000 associated deaths (1). Due to the widespread use of PSA screening, the mortality rate of the disease has declined by more than $50 \%$ (2). The results of the European Randomized Study of Screening Prostate Cancer revealed that a $20 \%$ reduction in mortality was attributable to PSA screening and treatment; however, 48 men had to be overtreated to prevent 1 cause-specific death from prostate cancer (3). Almost $60 \%$ of men who are diagnosed with prostate cancer may not require active therapy (4).

Active surveillance or watchful waiting (AS/WW) is an alternative to radical prostatectomy or radiotherapy, and for appropriately selected patients AS/WW can reduce overtreatment (5). The 2019 National Comprehensive Cancer Network (NCCN) Guidelines suggested that observation may be an option for men with low-risk or favorable intermediate-risk PCa (no more than 1 intermediate risk factor, ISUP grade $\leq 2$, and $<50 \%$ of biopsy cores positive) (6). A growing subset of prostate cancer patients are recognized to be candidates for AS/WW and AS/WW is increasingly being used (7-9). Nevertheless, the outcomes of observation in men with favorable intermediate-risk PCa are unclear and have produced mixed results $(10,11)$. A nomogram to guide the clinical selection of PCa patients who are suitable for AS/WW has yet to be developed.

To improve the prediction of prognosis for patients with ISUP grade 1 or 2 PCa who conform to the NCCN definition of low-risk PCa [prostate specific antigen $(\mathrm{PSA})<10 \mathrm{ng} / \mathrm{mL}$ and cT2 aN0M0 or less], we reviewed the information of patients registered in the Surveillance, Epidemiology, and End Results (SEER) Program from 2004 to 2015 and analyzed the association of tumor characteristics with prostate cancerspecific mortality (PCSM) and non-PCSM (considered a competing risk). We also constructed and validated a competing-risk nomogram to predict PCSM in order to assist clinical decision-making for PCa patients. We present the following article in accordance with the TRIPOD reporting checklist (available at http://dx.doi. org/10.21037/tau-20-994).

\section{Methods}

\section{Study patients}

Data of PCa cases diagnosed between 2004 to 2015 were extracted from the SEER Incidence database using the SEER ${ }^{*}$ Stat software (version 8.3.5) (https://seer.cancer.gov/ seerstat/software/). The inclusion criteria for cases were low-risk (LR) (ISUP $=1$ and PSA $<10 \mathrm{ng} / \mathrm{mL}$ and cT1$2 \mathrm{aNOM0}$ ) and selective intermediate-risk (SIR) (ISUP $=2$ and PSA $<10 \mathrm{ng} / \mathrm{mL}$ and cT1-2aN0M0) patients with AS/ WW. The exclusion criteria included: (I) incomplete clinical data; (II) patients with $>1$ primary cancer; (III) patients who received intervention treatments such as transurethral resection of the prostate, radical prostatectomy, radiotherapy, or chemotherapy; (IV) patients who were recommended treatment but refused it; (V) patients with uncertain cause of death. Prostate cancer-specific mortality was defined as death as a result of prostate. Competing mortality was defined as either non-prostate cancer mortality. For further analysis, age and PSA were used as continuous variables. Marital status was categorized as married or unmarried. Race was classified into white, black, or other. The clinical primary tumor category extension (cT) categories of the cases diagnosed between 2004-2009 were converted according to the American Joint Committee on Cancer (AJCC) $7^{\text {th }}$ edition [available on the SEER Registrar Staging Assistant website; https://staging.seer.cancer.gov/cs/ input/02.05.50/prostate/extension/?breadcrumbs $=(\sim s c h e m a$ list $),\left(\sim v i e w \_s c h e m a \sim, \sim\right.$ prostate $\left.\left.\sim\right)\right]$, in line with the cases between 2010-2015.

The study was conducted in accordance with the Declaration of Helsinki (as revised in 2013) and the Harmonized Tripartite Guideline for Good Clinical Practice from the International Conference on Harmonization. The data analyzed in this study are freely available from the SEER Incidence database (https://seer.cancer.gov/) and required no ethical approval.

\section{Statistical analysis}

The median follow-up values were the median observed survival time for cases between 2004-2015. For local PCa cases that had a low risk of clinical progression within $10-15$ years of diagnosis (12), the prognosis more vulnerable to competing events with increasing age, such as cardiovascular and cerebrovascular diseases. Therefore, the causes of death were divided into PCSM and non- 
Table 1 Cumulative incidences of death from prostate cancer and other causes with patient and tumor characteristics

\begin{tabular}{|c|c|c|c|c|c|c|c|c|c|}
\hline Characteristic & $\begin{array}{c}\text { No of } \\
\text { patients }\end{array}$ & \multicolumn{4}{|c|}{ Prostate cancer } & \multicolumn{4}{|c|}{ Other Causes } \\
\hline Race & & & & & 0.23 & & & & $<0.001$ \\
\hline Black & 5,241 & 71 & $0.92(0.60-1.22)$ & $2.82(1.97-3.58)$ & & 550 & 8.67 (7.44-9.16) & 22.35 (18.47-22.86) & \\
\hline Marital status & & & & & $<0.001$ & & & & $<0.001$ \\
\hline Married & 22,037 & 222 & $0.55(0.43-0.66)$ & 2.47 (2.06-2.83) & & 1,742 & $5.60(5.10-5.81)$ & $17.62(15.85-18.32)$ & \\
\hline $\mathrm{T} 2 \mathrm{a}$ & 1,875 & 25 & $0.91(0.38-1.42)$ & $3.49(1.86-5.0)$ & & 175 & $7.0(5.37-8.08)$ & $23.38(17.30-24.25)$ & \\
\hline ISUP grade & & & & & $<0.001$ & & & & $<0.001$ \\
\hline 1 & 25,376 & 240 & $0.53(0.42-0.63)$ & $2.14(1.79-2.45)$ & & 2,047 & $5.86(5.35-6.0)$ & $18.0(16.27-18.77)$ & \\
\hline 2 & 5,162 & 118 & $1.39(1.0-1.76)$ & 5.77 (4.40-6.79) & & 680 & $10.30(8.82-10.70)$ & $27.60(23.46-29.52)$ & \\
\hline
\end{tabular}

$\mathrm{Cl}$, confidence interval; cT stage, clinical T stage; ISUP, International Society of Urological Pathology.

PCSM, and the competing risk model was used for the analysis (13). The cumulative incidence function (CIF) was used to show the PCSM and non-PCSM for all patients and Gray's test was used to evaluate the difference (14). The PCSM and non-PCSM at 5 and 10 years were predicted with the Fine-Gray proportional hazards regression model. Subsequently, the cases were randomly divided into the training or validation cohort (1:1). A nomogram was constructed in the training cohort and validated using the validation cohort to visualize the competing risk models (15). Variables, including $\mathrm{c} T$ stage, race, age, marital status, PSA, and ISUP grade, which were significantly associated with outcomes were incorporated into the final nomogram. Finally, the performance of the model was evaluated through discrimination and calibration (16). Discrimination was defined to as the model's ability to identify events and was evaluated using the concordance index (C-index). The calibration curve was used to evaluate the agreement between the predictions of the model and observations using 500 bootstrap resamples.

All statistical analyses were performed using $\mathrm{R}$ software (version 3.6.1 software, https://www.r-project.org). The R packages "cmprsk", "rms", "mstate", and "pec" were used to model and develop the nomogram. A two-sided $\mathrm{P}$ value of $<0.05$ was considered to be statistically significant.

\section{Results}

\section{PCSM and competing risk analysis}

A total of 30,538 patients diagnosed from 2004 to 2015 on the SEER database were eligible for inclusion in our analysis. The detailed demographics and tumor characteristics of these patients are summarized in Table 1 .

The median follow-up of all patients was 60 months (range, 1-155 months). Among the 30,538 cases, there were $358(1.17 \%)$ deaths resulting from $\mathrm{PCa}$ and 2,727 $(8.93 \%)$ resulting from other causes. The 10 -year cumulative incidence of death from prostate cancer and death from other cause were $2.8 \%$ (95\% CI: $2.4-3.1 \%$ ) and 19.3\% (95\% CI: 17.8-20.5\%), respectively (Figure 1A). Among the other causes of death, the three most common causes were cardiac diseases (31.9\%), chronic obstructive pulmonary disease and associated conditions $(8.6 \%)$, and cerebrovascular disease (7.0\%). Table 1 summarizes the 5 - 

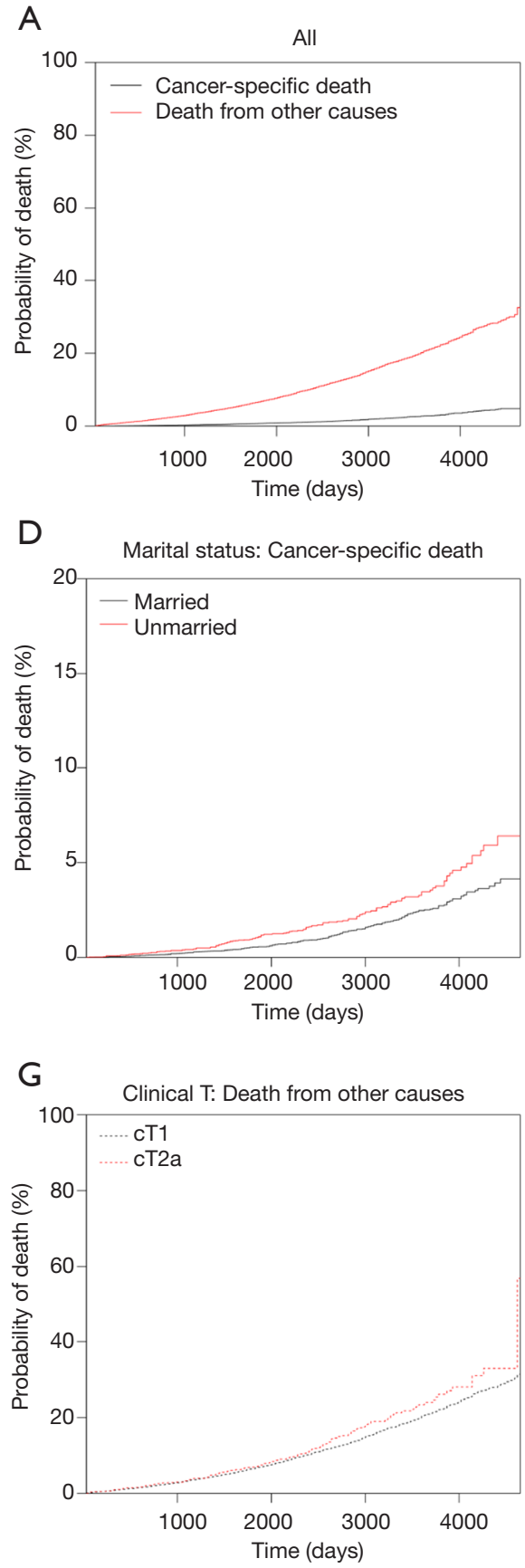
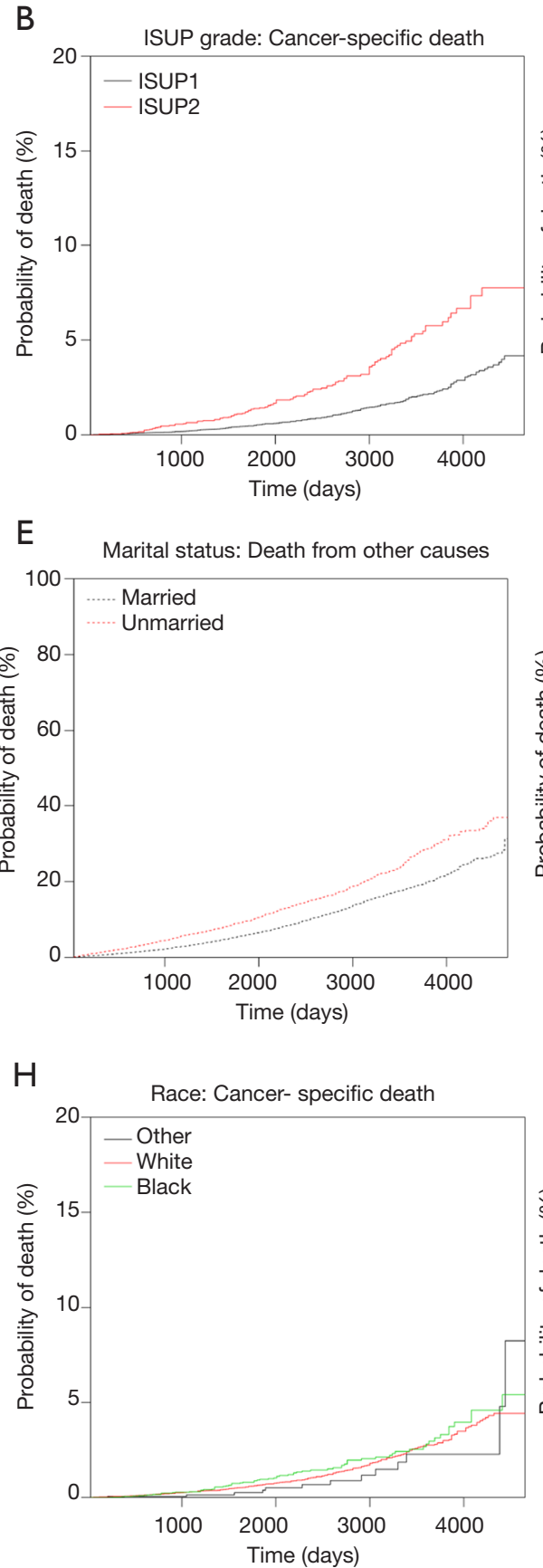
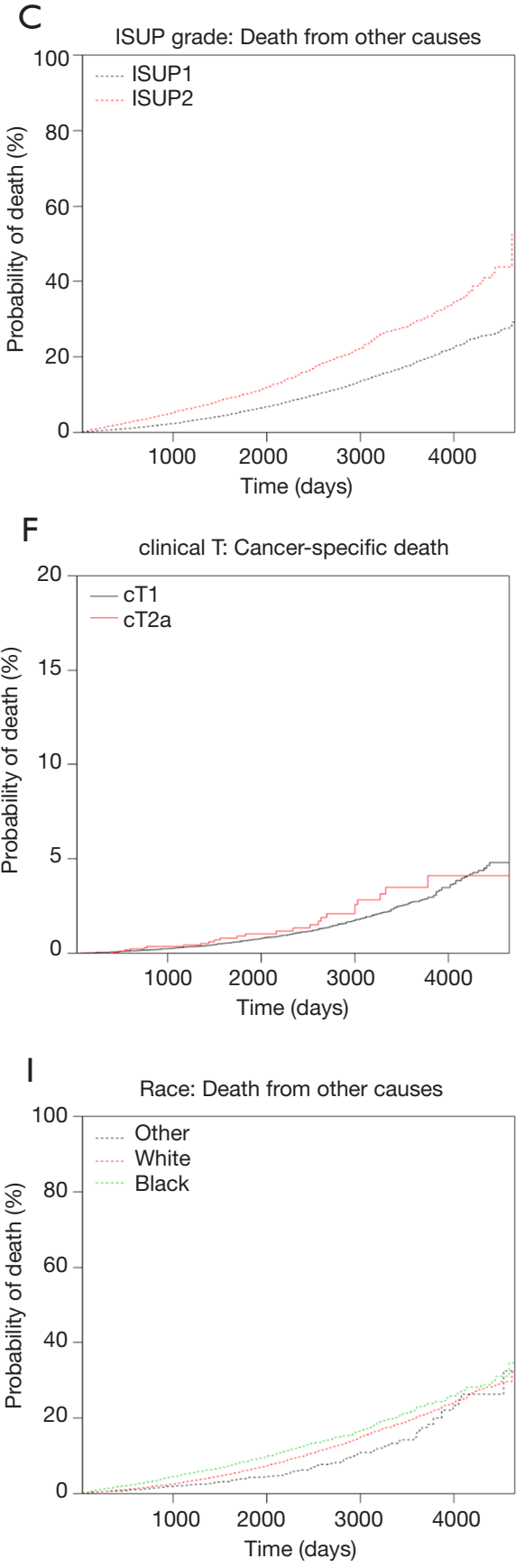

Figure 1 PCSM and competing risk analysis. (A) The probability of death from prostate cancer compared with that of death from other causes; (B,C,D,E,F,G,H,I) The probability of death from prostate cancer and other causes by ISUP grade, marital status, cT stage, and race. PCSM, prostate cancer-specific mortality; ISUP, International Society of Urological Pathology.

and 10-year PCSM and non-PCSM, together with patient and tumor characteristics. Figure 1 demonstrates the corresponding CIF curves.

Subsequently, competing risk analysis was performed to define whether the variables could predict the PCSM and non-PCSM. Statistically significant variables associated with PCSM in this model included age $(\mathrm{P}<0.001)$, marital status $(\mathrm{P}<0.001)$, PSA $(\mathrm{P}<0.001)$, and ISUP grade 
Table 2 Competing risk models of probabilities of death from prostate cancer and death from other causes

\begin{tabular}{|c|c|c|c|c|c|c|}
\hline \multirow{2}{*}{ Characteristics } & \multicolumn{3}{|c|}{ Death from prostate cancer } & \multicolumn{3}{|c|}{ Death from other causes } \\
\hline & Coefficient & sdHR $(95 \% \mathrm{Cl})$ & $P$ value & Coefficient & sdHR $(95 \% \mathrm{Cl})$ & $P$ value \\
\hline Other (reference) & & 1 & & & 1 & \\
\hline White & 0.27 & $1.32(0.75-2.30)$ & 0.33 & 0.30 & $1.35(0.97-1.36)$ & 0.002 \\
\hline \multicolumn{7}{|l|}{ Age (year) } \\
\hline $\mathrm{Age}^{*}$ & 0.063 & 1.07 (1.05-1.08) & $<0.001$ & 0.095 & $1.10(1.09-1.11)$ & $<0.001$ \\
\hline \multicolumn{7}{|l|}{ Marital status } \\
\hline cT1 (reference) & & 1 & & & 1 & \\
\hline cT2a & 0.18 & $1.20(0.79-1.81)$ & 0.38 & 0.01 & $1.02(0.87-1.19)$ & 0.82 \\
\hline \multicolumn{7}{|l|}{ PSA (ng/mL) } \\
\hline $\mathrm{PSA}^{*}$ & 0.094 & $1.10(1.04-1.16)$ & $<0.001$ & 0.054 & $1.06(1.03-1.08)$ & $<0.001$ \\
\hline \multicolumn{7}{|l|}{ ISUP grade } \\
\hline 1 (reference) & & 1 & & & 1 & \\
\hline 2 & 0.64 & $1.89(1.51-2.37)$ & $<0.001$ & 0.21 & $1.23(1.13-1.35)$ & $<0.001$ \\
\hline
\end{tabular}

$(\mathrm{P}<0.001)$. PCSM was higher among older patients, with a sub-distribution hazard ratio (sdHR) of 1.07 (95\% CI: 1.05-1.08). Every extra unit of PSA was associated with a significant increase in PCSM, with an sdHR of $1.10(95 \%$ CI: 1.04-1.20). Patients with ISUP 2 were more likely to die of PCa (sdHR =1.89, 95\% CI: 1.51-2.37). Unmarried patients had a higher PCSM than those who were married (sdHR =1.61, 95\% CI: 1.29-2.0). Black patients had higher PCSM than patients who were white or of other races, but there were no significant differences. Patients with cT2a had a higher PCSM than cT1 patients, but there were no significant differences. Similarly, older age, unmarried status, white and black race, higher PSA, and higher ISUP grade increased non-PCSM; however, cT2a patients had higher non-PCSM than cT1 patients, although there were no significant differences (Table 2).

\section{PCSM nomogram}

All patients were randomly divided into the training or the validation cohort (1:1). The detailed demographics and tumor characteristics of the two cohorts are summarized in Table S1. A nomogram to predict the probability of PCSM at 5, 10, and 12 years was constructed in the training cohort, based on age, PSA, marital status, and ISUP grade (Figure 2). The nomogram had a reliable performance in predicting PCSM, with a C-index of 0.744 (95\% CI: 0.700-0.781, $\mathrm{P}<0.001)$. The calibration curve based on the training cohort showed good agreement between prediction and observation in 5-, 10-, and 12-year PCSM (Figure 3A). Similarly, in the validation cohort, the nomogram indicated excellent accuracy in predicting PCSM, with a C-index of 0.738 (95\% CI: $0.700-0.777, \mathrm{P}<0.001$ ). The calibration curve also showed that the nomogram had an excellent 


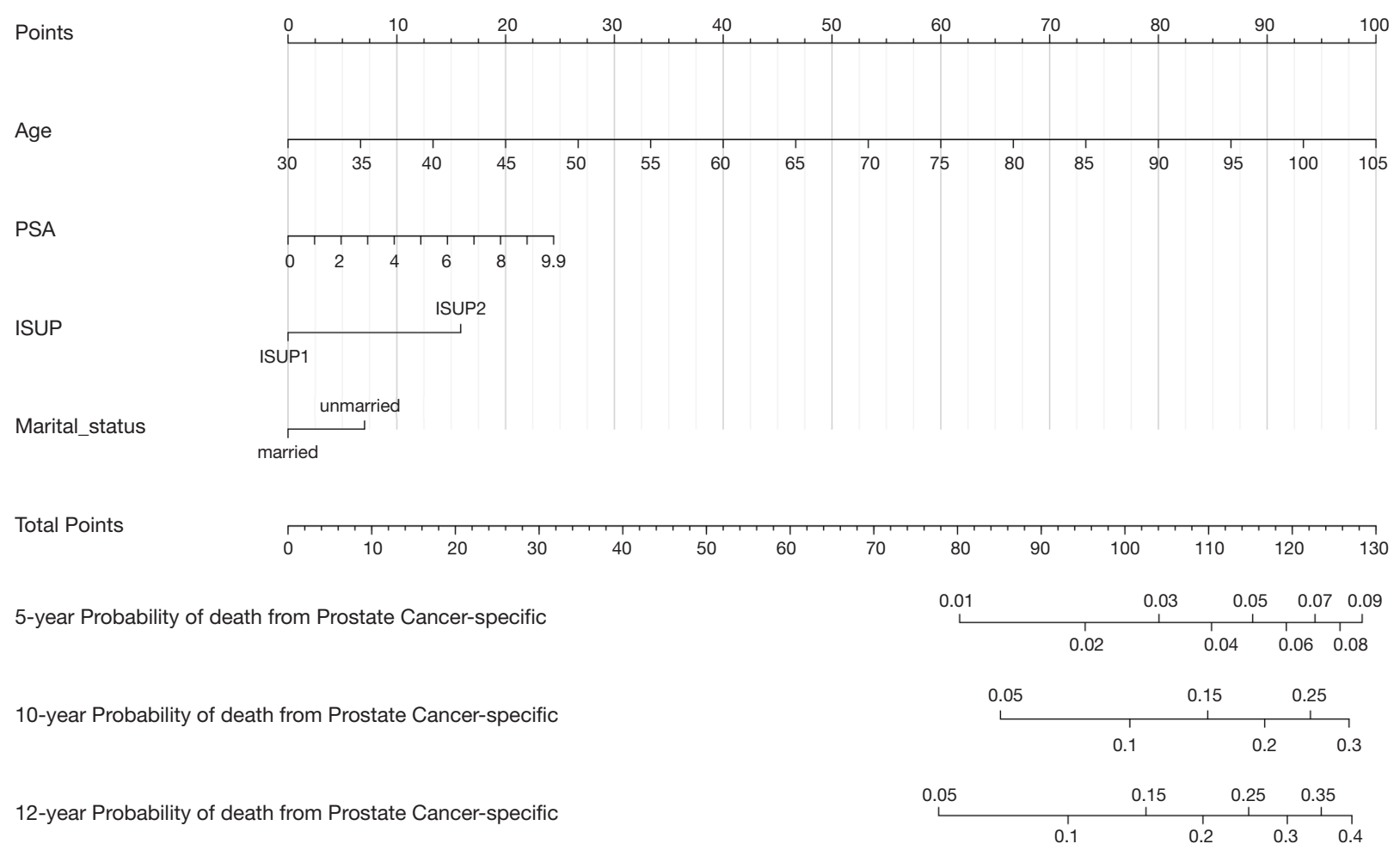

Figure 2 Nomogram to predict the 5-, 10-, and 12-year PCSM of LR and SIR prostate cancer patients with AS/WW. PCSM, prostate cancer-specific mortality; LR, low-risk; SIR, selective intermediate-risk; PSA, prostate specific antigen; ISUP, International Society of Urological Pathology.

performance in predicting PCSM in the validation cohort (Figure 3B).

\section{Discussion}

The challenges associated with the application of PSA have emphasized the need to develop more objective measures for identifying clinically significant PCa while continuing to reduce the sequence of over-diagnosis and over-treatment (17). In randomized controlled trials, AS/ WW has been proven as an alternative clinical strategy to active treatment for appropriately selected patients $(18,19)$. A number of research about AS/WW have reported favorable short- to medium- term outcomes among lowrisk PCa patients, including cohorts from Johns Hopkins University (9), Memorial Sloan-Kettering Cancer Center (20), the Royal Marsden Hospital (21), University of California, San Francisco $(22,23)$, and the University of Toronto (24).

However, most studies to date have focused on AS/WW for low-risk or intermediate-risk PCa patients whose life expectancy is less than 10 years. Moreover, the prognoses of intermediate-risk PCa patients with AS/WW are complex and varied $(18,25)$ due to different inclusion criteria and the lack of a reliable PCSM Nomogram. The University of Toronto cohort included a total of 450 patients with AS, $14 \%$ with PSA higher than $10 \mathrm{ng} / \mathrm{mL}, 17 \%$ with ISUP2 and ISUP3, and 3\% with both risk factors (24). However, the results did not accurately identify highrisk patients who were not suitable for AS. In the study of Cooperberg et al., PCa patients with AS were classified as low- or intermediate-rise based on the UCSF Cancer of the Prostate Risk Assessment (CAPRA) score. And they found that part of intermediate-risk patients may be appropriate candidates for AS (23). Similarly, Musunuru et al. included LR patients and part of intermediaterisk patients (age $>70$ years and cT2c or PSA $\leq 15 \mathrm{ng} / \mathrm{mL}$ ). Their results showed that LR and intermediate-risk patients with ISUP1 could receive AS, but not for ISUP2 PCa (26). Ploussard et al. also tried to explore the inclusion criteria 

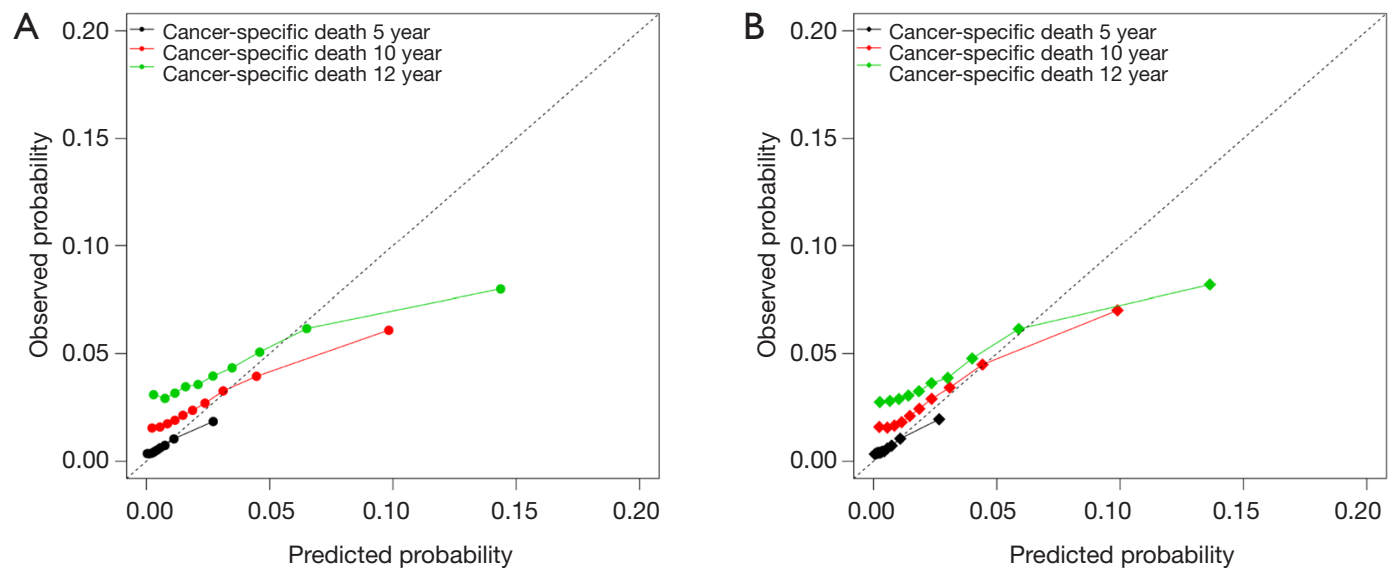

Figure 3 Calibration plot of the nomogram. (A) Calibration plot in the training cohort; (B) Calibration plot in the validation cohort. The dotted line represents equality between the predicted and observed probabilities.

of AS and conducted a retrospective analysis including 2,323 patients with localized ISUP2 PCa. Their research suggests that patients with ISUP2 PCa could receive AS but should adhere to strict selection criteria (10). Thus, development of a nomogram may be especially beneficial for select which patients to receive AS.

Further, the 2019 NCCN Guidelines recommend AS/ $\mathrm{WW}$ as an option for patients with favorable intermediaterisk $\mathrm{PCa}$ (no more than 1 intermediate risk factor, ISUP grade $\leq 2$, and $<50 \%$ of biopsy cores positive) (6). Therefore, we included LR and SIR PCa patients with AS/WW in our study to predict their prognosis and to provide some guidance for clinical trials. A nomogram to predict PCSM of LR and SIR patients with AS/WW was constructed and validated. To our knowledge, the current study is the first report of AS/WW use for patients with LR and SIR prostate cancer to be based on a large populationbased database across the United States. Therefore, the nomogram we designed is the first to be used to identify patients potentially suitable for AS/WW and for the design of clinical trials involving intermediate risk patients with AS/WW.

We found that patients with SIR ISUP2 had higher PCSM than those with LR ISUP1 after adjusting for PSA, cT stage, race, and marital status. This result is consistent with previous findings that SIR PCa patients with AS are more likely than LR patients to upgrade to unfavorable disease (27). In addition, Raldow et al. found that favorable intermediate risk PCa did not have significantly increased risk of PCSM compared with low-risk PCa following radiotherapy and ISUP grade did not have statistical significance for PCSM (28). It may reveal that favorable intermediate risk $\mathrm{PCa}$ could benefit from radiotherapy. However, Butler et al. reported that PCSM of favorable intermediate risk PCa have no statistical difference between radical prostatectomy/radiotherapy and AS/WW (28). To sum up, it implies that AS/WW treatment for SIR patients is feasible, but should be followed more closely. Furthermore, most evidence for the application of AS/ WW in SIR patients has come from retrospective data. Therefore, prospective trials are needed to further evaluate the safety of AS/WW for SIR patients.

Between 2010 and 2015, the number of black and nonblack patients with AS/WW increased (29). Our findings show that the difference in PCSM between black and white patients was not statistically significant. Previous studies based on the SEER database from 2010-2015 show that PCSM was significantly higher for black patients with lowgrade ISUP1 who underwent AS/WW than their non-black counterparts (30); however, the median follow-up in Mahal et al.'s study was 36 months, which was shorter than our study's median follow-up time of 60 months. Therefore, we infered that racial disparities might not exist among patients who receive AS/WW.

In accordance with previous studies, our data also showed that high PSA was associated with an increased risk of PCSM among PCa patients with AS/WW (26,31). Furthermore, patients who were unmarried had higher risks of PCSM and non-PCSM than those who were married, and this finding is supported by multiple retrospective reviews $(32,33)$. Overall, our nomogram was consistent with the results of previous studies, indicating its reliability 
and helpfulness in predicting the prognosis of $\mathrm{PCa}$ with AS/WW.

We noted that older age had a negative impact on PCSM in patients with AS/WW (sdHR $=1.07,95 \%$ CI: 1.05-1.08), and the impact on non-PCSM was even more significant (sdHR $=1.10,95 \%$ CI: 1.09-1.11), which was consistent with the findings of previously published literature (33-35). This result implies that with increasing age, non-PCSM becomes higher than that of PCSM. Therefore, the possibility of non-PCSM should be taken into consideration during clinical decision-making for elderly patients. Younger patients are therefore suitable for AS/WW as they have lower PCSM, but need longer follow-up times.

Our study has several limitations. Firstly, the analyses of data were retrospective and heterogeneous. Secondly, the SEER database cannot discriminate between AS and WW. Thirdly, the SEER database lacks information, such as comorbidities, subsequent treatments, and the percentage of Gleason pattern 4 in specimens. Additionally, the SEER database does not contain information on the number of positive cores, which is an important inclusion criterion for AS/WW. We believe that our inclusion criteria (ISUP $\leq 2$ and PSA $<10 \mathrm{ng} / \mathrm{mL}$ and cT1-2aNOM0) are by and large opposite to the concept of clinically significant disease based on expression of "definition one" (36). It also conforms to the criteria of favorable intermediate-risk PCa defined in the 2019 NCCN Guidelines (6).

In conclusion, the prognosis of LR and SIR PCa patients with AS/WW was excellent. Our competing risk nomogram showed a good performance in predicting PCSM. It could serve as a useful clinical tool for identifying patients with higher risk of PCSM and selecting candidates for AS/WW.

\section{Acknowledgments}

Funding: This study was supported by grants from National Natural Science Foundation of China (No.81600542 and 81670643); the Natural Science Foundation of Guangdong Province (2020A1515010464); the Guangdong Basic and Applied Basic Research Foundation (Grant NO. 2019A1515110033); Distinguished Young Talents in Higher Education Foundation of Guangdong Province (Grant NO. 2019KQNCX115 and 2020KZDZX1168); China Postdoctoral Science Foundation (Grant NO. 2019M662865); Achievement cultivation and clinical transformation application cultivation projects of the First Affiliated Hospital of Guangzhou Medical University (Grant NO. ZH201908).

\section{Footnote}

Reporting Checklist: The authors have completed the TRIPOD reporting checklist. Available at http://dx.doi. org/10.21037/tau-20-994

Conflicts of Interest: All authors have completed the ICMJE uniform disclosure form (available at http://dx.doi. org/10.21037/tau-20-994). The authors have no conflicts of interest to declare.

Ethical Statement: The authors are accountable for all aspects of the work in ensuring that questions related to the accuracy or integrity of any part of the work are appropriately investigated and resolved. The study was conducted in accordance with the Declaration of Helsinki (as revised in 2013) and the Harmonized Tripartite Guideline for Good Clinical Practice from the International Conference on Harmonization. The data analyzed in this study are freely available from the SEER Incidence database (https://seer.cancer.gov/) and required no ethical approval.

Open Access Statement: This is an Open Access article distributed in accordance with the Creative Commons Attribution-NonCommercial-NoDerivs 4.0 International License (CC BY-NC-ND 4.0), which permits the noncommercial replication and distribution of the article with the strict proviso that no changes or edits are made and the original work is properly cited (including links to both the formal publication through the relevant DOI and the license). See: https://creativecommons.org/licenses/by-nc-nd/4.0/.

\section{References}

1. Fitzmaurice C, Abate D, Abbasi N, et al. Global, Regional, and National Cancer Incidence, Mortality, Years of Life Lost, Years Lived With Disability, and DisabilityAdjusted Life-Years for 29 Cancer Groups, 1990 to 2017: A Systematic Analysis for the Global Burden of Disease Study. JAMA Oncol 2019;5:1749-68.

2. Catalona WJ. Prostate Cancer Screening. Med Clin North Am 2018;102:199-214.

3. Schröder FH, Hugosson J, Roobol MJ, et al. Screening and prostate-cancer mortality in a randomized European study. N Engl J Med 2009;360:1320-8.

4. Welch HG, Black WC. Overdiagnosis in cancer. J Natl Cancer Inst 2010;102:605-13.

5. Kinsella N, Stattin P, Cahill D, et al. Factors Influencing 
Men's Choice of and Adherence to Active Surveillance for Low-risk Prostate Cancer: A Mixed-method Systematic Review. Eur Urol 2018;74:261-80.

6. Mohler JL, Antonarakis ES, Armstrong AJ, et al. Prostate Cancer, Version 2.2019, NCCN Clinical Practice Guidelines in Oncology. J Natl Compr Canc Netw 2019;17:479-505.

7. Womble PR, Montie JE, Ye Z, et al. Contemporary use of initial active surveillance among men in Michigan with low-risk prostate cancer. Eur Urol 2015;67:44-50.

8. Mahal BA, Butler S, Franco I, et al. Use of Active Surveillance or Watchful Waiting for Low-Risk Prostate Cancer and Management Trends Across Risk Groups in the United States, 2010-2015. JAMA 2019;321:704-6.

9. Carter HB, Walsh PC, Landis P, et al. Expectant management of nonpalpable prostate cancer with curative intent: preliminary results. J Urol 2002;167:1231-4.

10. Ploussard G, Isbarn H, Briganti A, et al. Can we expand active surveillance criteria to include biopsy Gleason $3+4$ prostate cancer? A multi-institutional study of 2,323 patients. Urol Oncol 2015;33:71.e1-9.

11. Yamamoto T, Musunuru HB, Vesprini D, et al. Metastatic Prostate Cancer in Men Initially Treated with Active Surveillance. J Urol 2016;195:1409-14.

12. Thomsen FB, Brasso K, Klotz LH, et al. Active surveillance for clinically localized prostate cancer--a systematic review. J Surg Oncol 2014;109:830-5.

13. Austin PC, Lee DS, Fine JP. Introduction to the Analysis of Survival Data in the Presence of Competing Risks. Circulation 2016;133:601-9.

14. Wolbers M, Koller MT, Stel VS, et al. Competing risks analyses: objectives and approaches. Eur Heart J 2014;35:2936-41.

15. Iasonos A, Schrag D, Raj GV, et al. How to build and interpret a nomogram for cancer prognosis. J Clin Oncol 2008;26:1364-70.

16. Harrell FE, Lee KL, Mark DB. Multivariable prognostic models: issues in developing models, evaluating assumptions and adequacy, and measuring and reducing errors. Stat Med 1996;15:361-87.

17. Loeb S, Bjurlin MA, Nicholson J, et al. Overdiagnosis and overtreatment of prostate cancer. Eur Urol 2014;65:1046-55.

18. Wilt TJ, Brawer MK, Jones KM, et al. Radical prostatectomy versus observation for localized prostate cancer. N Engl J Med 2012;367:203-13.

19. Hamdy FC, Donovan JL, Lane JA, et al. 10-Year Outcomes after Monitoring, Surgery, or Radiotherapy for Localized Prostate Cancer. N Engl J Med 2016;375:1415-24.

20. Patel MI, DeConcini DT, Lopez-Corona E, et al. An analysis of men with clinically localized prostate cancer who deferred definitive therapy. J Urol 2004;171:1520-4.

21. Ulmert D, Serio AM, O'Brien MF, et al. Long-term prediction of prostate cancer: prostate-specific antigen (PSA) velocity is predictive but does not improve the predictive accuracy of a single PSA measurement 15 years or more before cancer diagnosis in a large, representative, unscreened population. J Clin Oncol 2008;26:835-41.

22. Dall'Era MA, Konety BR, Cowan JE, et al. Active surveillance for the management of prostate cancer in a contemporary cohort. Cancer 2008;112:2664-70.

23. Cooperberg MR, Cowan JE, Hilton JF, et al. Outcomes of active surveillance for men with intermediate-risk prostate cancer. J Clin Oncol 2011;29:228-34.

24. Klotz L, Zhang L, Lam A, et al. Clinical results of longterm follow-up of a large, active surveillance cohort with localized prostate cancer. J Clin Oncol 2010;28:126-31.

25. Bill-Axelson A, Holmberg L, Garmo H, et al. Radical prostatectomy or watchful waiting in early prostate cancer. N Engl J Med 2014;370:932-42.

26. Musunuru HB, Yamamoto T, Klotz L, et al. Active Surveillance for Intermediate Risk Prostate Cancer: Survival Outcomes in the Sunnybrook Experience. J Urol 2016;196:1651-8.

27. Gearman DJ, Morlacco A, Cheville JC, et al. Comparison of Pathological and Oncologic Outcomes of Favorable Risk Gleason Score $3+4$ and Low Risk Gleason Score 6 Prostate Cancer: Considerations for Active Surveillance. J Urol 2018;199:1188-95.

28. Raldow AC, Zhang D, Chen MH, et al. Risk Group and Death From Prostate Cancer: Implications for Active Surveillance in Men With Favorable Intermediate-Risk Prostate Cancer. JAMA Oncol 2015;1:334-40.

29. Butler S, Muralidhar V, Chavez J, et al. Active Surveillance for Low-Risk Prostate Cancer in Black Patients. N Engl J Med 2019;380:2070-2.

30. Mahal BA, Berman RA, Taplin ME, et al. Prostate CancerSpecific Mortality Across Gleason Scores in Black vs Nonblack Men. JAMA 2018;320:2479-81.

31. Klotz L, Vesprini D, Sethukavalan P, et al. Long-term follow-up of a large active surveillance cohort of patients with prostate cancer. J Clin Oncol 2015;33:272-7.

32. Loeb S, Byrne N, Makarov DV, et al. Use of Conservative Management for Low-Risk Prostate Cancer in the Veterans Affairs Integrated Health Care System From 
2005-2015. JAMA 2018;319:2231-3.

33. Knipper S, Pecoraro A, Palumbo C, et al. A 25-year Period Analysis of Other-cause Mortality in Localized Prostate Cancer. Clin Genitourin Cancer 2019;17:395-401.

34. Butler SS, Mahal BA, Lamba N, et al. Use and early mortality outcomes of active surveillance in patients with intermediate-risk prostate cancer. Cancer 2019;125:3164-71.

35. Abdollah F, Sun M, Schmitges J, et al. Cancer-specific and other-cause mortality after radical prostatectomy versus

Cite this article as: $\mathrm{Wu} \mathrm{X}, \mathrm{Lv} \mathrm{D}$, Eftekhar M, Cai C, Zhao Z, Gu D, Liu Y. Cause-specific mortality of low and selective intermediate-risk prostate cancer patients with active surveillance or watchful waiting. Transl Androl Urol 2021;10(1):154-163. doi: 10.21037/tau-20-994 observation in patients with prostate cancer: competingrisks analysis of a large North American population-based cohort. Eur Urol 2011;60:920-30.

36. Simmons LAM, Ahmed HU, Moore CM, et al. The PICTURE study -- prostate imaging (multi-parametric MRI and Prostate HistoScanning ${ }^{\mathrm{TM}}$ ) compared to transperineal ultrasound guided biopsy for significant prostate cancer risk evaluation. Contemp Clin Trials 2014;37:69-83. 


\section{Supplementary}

Table S1 Patients characteristics of training and validation cohorts

\begin{tabular}{|c|c|c|c|c|}
\hline Characteristics & All patients & Training & Validation & $P$ value \\
\hline Age, average years (range) & 65 (34-105) & $65(35-105)$ & 65 (34-99) & 0.42 \\
\hline PSA, average ng/mL (range) & $5.7(0.1-9.9)$ & $5.7(0.1-9.9)$ & $5.7(0.1-9.9)$ & 0.64 \\
\hline Race, no, (\%) & & & & 0.59 \\
\hline White & $23,686(77.6)$ & $11,846(77.6)$ & $11,840(77.5)$ & \\
\hline Black & $5,241(17.1)$ & 2,637 (17.2) & $2,604(17.1)$ & \\
\hline Marital status, no., (\%) & & & & 0.37 \\
\hline Married & $22,037(72.2)$ & $10,984(71.9)$ & $11,053(72.4)$ & \\
\hline T1c & $28,663(93.9)$ & $14,361(94.0)$ & $14,302(93.7)$ & \\
\hline $\mathrm{T} 2 \mathrm{a}$ & $1,875(6.1)$ & $909(6.0)$ & $966(6.3)$ & \\
\hline ISUP grade, no., (\%) & & & & 0.88 \\
\hline 1 & $25,376(83.1)$ & $12,683(83.1)$ & $12,693(83.1)$ & \\
\hline 2 & $5,163(16.9)$ & $2,587(16.9)$ & $2,576(16.9)$ & \\
\hline Cause of death, no., (\%) & & & & 0.91 \\
\hline prostate cancer & $358(11.6)$ & $176(11.5)$ & $182(11.7)$ & \\
\hline other causes & $2,727(88.4)$ & $1352(88.5)$ & $137(88.3)$ & \\
\hline
\end{tabular}

PSA, prostate specific antigen; cT stage, clinical T stage; ISUP, International Society of Urological Pathology. P value: Student's t test and Chi-square test was used to test whether there was any difference in clinical characteristics between the training and validation cohorts. 